\title{
An Acoustics Intensity Based Investigation of the Energy Flow Over the Barriers
}

\author{
S. WEYNA* \\ West Pomeranian University of Technology, Poland
}

\begin{abstract}
Many of theoretical research of the acoustics fields provides useful information about pressure fields, but none currently offers a full mapping of the acoustic energy flow (vectorial effects) in front and back of any scattering systems working in 3D real environmental conditions. Interference, diffraction and scattering of waves made the real field very complex and difficult to the theoretical modelling. This is one of the reasons why the experimental investigations of acoustic fields using sound intensity (SI) technique are so effective and serviceable methods. The visualization of acoustic energy flow in real-life acoustic 3D space fields can explain many particulars energetic effects (perturbations and vortex flow, effects of scattering in direct and near field, etc.), concerning the areas in which it is difficult to make numerical modeling and analysis with the CFD-FSI-CAA simulation methods. The sound intensity image represents a more accurate and efficient information compare to the spatial sound fields modelled. The article presents the application of SI technique to graphic presentation of spatial distribution the acoustic energy flow over the barriers of various geometrical shapes structures located in a three-dimensional space. As the results of research, the graphic analysis of the sound intensity flux in $2 \mathrm{D}$ and $3 \mathrm{D}$ space is show. Visualisation of research results is shown in the form of intensity streamlines in space and as a shape of flow wave or isosurface in three-dimensional space. Numerous examples illustrate the application of the SI measurement for practical problems at the vibroacoustical diagnostic and noise abatement, as well as to the validation of results of CFD/CAA numerical modelling. The differences, if appearance, mainly result from the fact that theoretical forecasting uses far too big simplifications or that it is impossible to obtain proper data on real physical features of the tested area and structures.
\end{abstract}

PACS numbers:

\section{Introduction}

It is widely accepted that the pulsating pressure acoustic wave, excites the structure, which then radiates sound power to the surrounding environment, but the mechanism of noise-generated phenomenon is not jet completely understood. The structural deformation causes fluid nodes to move, making it difficult to achieve a converged solution. Radiated noise is determined from the surface deformation using vibration-acoustics.

Modeling the wave impingement noise is non-trivial exercise. It requires a numerical simulation with FSI (Fluid Structure Interaction) methods. Many times, sufficient resolution of the local turbulence, complicated by the varying gas temperature and often complex geometry is necessary. Modeling this phenomenon requires representation of both the fluid and structure elements as well as their interaction. The feasibility of modeling this phenomenon can be done using CFD (Computational Fluid Dynamics), a commercial code with both FSI and CAA (Computational Aero-Acoustic). Both fluid and structural dynamics need to be modeled and are described by different sets of equations. Once the flow and struc-

* corresponding author; e-mail: weyna@ps.pl ture interaction was solved, the surface velocity at the structure can be post processed to calculate the sound pressure level at any predefined locations.

But we must remember that the numerical modeling should be validate using experimental investigation on the properly model or the real structure. In this paper, suggested validation possibility has been investigated. Sound intensity measurement results on a few models located in a semi-anechoic room may be compared with the prediction results. After investigations, details of the flow field and the interaction between fluid and structure will be a graphically visualized with proposed own methods and software.

The verifying tests using an intensity technique may show how much a theoretical image of an acoustic field distribution is differing from the distributions obtained through the measurements in real conditions. The degree of discrepancy between the predicted numerical results and the real structure of the field formed over barriers, grows proportionally to the degree to which the simplified calculated assumptions differ from the conditions encountered in reality. The differences mainly result from either the fact that theoretical forecasting uses too sweeping simplifications or that it is impossible to obtain proper data on real physical features of the tested area. This is one of the causes why the experimental 
investigation fields using the sound intensity technique differ according to computer simulating with $\mathrm{CFD} / \mathrm{CAA}$ methods.

\section{Sound intensity and flow visualizations}

The sound intensity measurements has become one of the most interesting techniques employed in solving vibroacoustic problems as well as in acoustic metrology, which simplifies the technique of measurements thus effectively replacing classical methods. Flow motion as the acoustic particle velocity may be measured experimentally using sound intensity probe, which can be used to collect the data to visualisation all the phenomena occurring in investigated acoustic vector fields, even in the near field and three dimensional space. The visualization of acoustic energy flow in real-life acoustic 3D space fields can be explain many particulars energetic effects (scattering, vortex flow, shielding area, etc.), concerning the areas in which it is difficult to make numerical modeling and analysis with the CFD-FSI-CAA methods [1-3].

Energy distribution images in acoustic fields, connected with the graphical presentation of the flow waves are a new element in acoustic metrology. Introduction of these possibilities have greatly changed the approach to examining many acoustic phenomena. This sound intensity measurement technique has been use to various studies on theoretical and applied acoustics, greatly simplifying the methods of research [4]. This is because it does not require criteria as strict as in traditional measurements, and the precision of direct measurements in real-life situations does not vary from laboratory experiments. The measurements can be carried out in a near field and in the fields with presence of parasite noise, which is a significant advantage in research. In the paper, experimental studies will be mode on real models and structures and documented with graphical records as the effects of wave interference of acoustic fields created around the obstacles and barriers placed in the flow field.

In the paper author have described the visualization methods in acoustic flow fields and show how these methods may assist scientists to gain understanding of complex acoustic energy flow in real-life field. A graphical method will be presented to determine the real acoustic wave distribution in 3D flow field. Visualizations of research results are shown in the form of intensity streamlines in space and as a shape of floating acoustic wave or as the intensity isosurface in three-dimensional space, which is unavailable to done by conventional acoustics metrology. In traditional acoustic metrology, the analysis of acoustic fields concerns only the distribution of pressure levels (scalar variable), however in a real acoustic field both scalar (acoustic pressure) and vector (the acoustic particle velocity) effects are closely related. Only when the acoustic field is described by the energy effect both potential and kinetic, may we understand the mechanisms of propagation, diffraction and scattering of acoustic flow waves. Application of the sound intensity method, including the presentation of space vector distribution of acoustic power, may bring new insight into the nature of acoustic field formation in real conditions of working sources [5]. This attribute of intensity method is very important in any industry acoustic investigations.

Based on the research with intensity technique and using selected visualizations methods, in the publication are demonstrating few examples of vector space distribution of the real acoustic field, illustrate the application of the SI measurement for practical problems at the acoustical diagnostic and noise abatement. Analysis of the results makes it possible to obtain much new information about energetic and geometric distributions of the acoustic fields [6]. The measurement technique described, as well as the method of graphical presentation of results, can enrich the knowledge of the mechanism of acoustic energy flux through the real partitions.

\section{Experimental study of the wave fields}

In an effort to understand the behavior and validate the performance of a split hydrodynamic-acoustic computational aeroacoustics approach coupled with a reflecting boundary condition, a series of experimental investigations are presented in this work. Validation case considered includes the classic aeroacoustic problem of the turbulent spinning vortex pair in near field, and the prediction of sound generation in a shear layer or in the far-field region.

\subsection{Introduction to acoustic fluid-structure interaction}

Acoustic of Fluid-Structure Interactions (FSI) includes the theory of aerodynamics sound, as well as more conventional areas of acoustics and structural vibration. The interactions between flow and structure are strong and flow-induced forces on bodies and structures include vibration. This vibration, in turn, includes additional noise and may even modify the unsteady fluid flow. In many cases, the structural surfaces do vibrate, but these vibrations cannot alter the flow motion.

When sound waves, propagating in a uniform medium, encounter a foreign object, the sound is scattered in all directions [7]. If the size of the object is about the same size as the wavelength of the sound, the scattering may be called strong and its pattern may be complicated. If the geometry of the object were relatively simple, it would be possible to calculate the scattered sound field. However, if there is a second object, located a few wavelengths away from the first object, then the scattered sound may reflect back and forth between the objects, undergoing multiple scattering.

To determine the sound generation by fluid structure interactions, it is necessary to know the response of the boundary to the turbulence stress [8]. These stresses not only generate sound but also excite structural vibrations that can store a significant amount of flow energy. Frictional forces ultimately dissipate the vibrations, but 
they can contribute substantially to the radiated noise because elastic waves are scattered at structural discontinuities, and some of their energy is transformed into sound. Thus, flow-generated sound reaches the far field via two paths: directly from the turbulence sources and indirectly from possible remote locations where the scattering occurs. Typical examples include the turbulent flow an aircraft and ship hulls, from duct flows, piping systems, and turbo machines.

A generally used method for the prediction of aerodynamic sound simulation is the coupling of the near-field solution of unsteady fluid flows and the far-field solution of the sound propagation/radiation. Many researchers for a range of aeroacoustic problems have adopted such a coupling strategy, and some good predictions have been obtained. The near field solution must identify the sources of sound, due to turbulence, the interaction between vortex structures and embedded solid surface, etc. Once these sources have been identified and extracted, an optimized high-order numerical scheme with minimized dissipation and dispersion features may be employed to calculate the propagation of the sound. Nevertheless, the efficient and accurate evaluation of the near-field sound sources still remains an open and challenging problem. Ever since Lighthill [9] proposed the first aerodynamic sound theory, most work in the computation of aeroacoustic problems is based on various acoustic analogies. The modeling of acoustic sources adopts certain basic source types, including monopoles, dipoles and quadrupoles, or their combinations, of which source strengths are known a priori, or these sources are directly calculated using the Lighthill stress tensor. In many situations the flow of interest is both unsteady and turbulent, and one needs to solve the full unsteady flow problem in order to adequately describe the hydrodynamic field and determine the sources of sound.

\subsection{Wave propagation in arrays of scatterers region}

The general scope of this research is to extend the knowledge of CAA to subsonic confined flows and gain more insight in the aero-acoustic behavior of such systems. In order to obtain this, the article presents the application of sound intensity technique to graphic presentation of spatial distribution of the acoustic vectors field. As the results of research a two and three-dimensional flow map of time-averaged active intensity vector in space will show. Additionally, the presentation of the distribution of the intensity streamlines around the barriers was attached.

For the sound intensity measurement the 3D-USP type miniature intensity probe is applied. It is a very compact and integrated sound intensity transducer combines three orthogonal positioned particle velocity sensors and a miniature ( 0.1 inch) pressure microphone. The actual $3 \mathrm{D}$ sensor configuration without its cap is less than $5 \mathrm{~mm} \times 5 \mathrm{~mm} \times 5 \mathrm{~mm}$.

In the first part of investigation, the tests concern the application of sound intensity technique to graphic presentation of spatial distribution the acoustic energy flow around free hanged rectangular plate with dimension of $0.6 \mathrm{~m} \times 03 \mathrm{~m}$ and thickness $6 \mathrm{~mm}$, located in a anechoic chamber and excited by axially travelling incident wave (stationary broad band pink noise) coming from loudspeaker on a distance $0.6 \mathrm{~m}$ central, before to the plate. The measurements with using intensity probe are curry out in one-twelfth octave bands in the frequency range between $25 \mathrm{~Hz}$ and $6300 \mathrm{~Hz}$.

Visualization of the results of measurements are illustrated in Fig. 1 as a sound intensity distributions map in plane of axis symmetry, as intensity streamlines in space before the plate, as a shape of floating acoustic wave (as well as the "velocity profile" in the fluid mechanics), and as a shape of sound intensity isosurface. On the Fig. 1, the results of sound intensity components measured on complete 3D space around obstacle, are show here only in half measured space.

Direct measurement of the acoustic power flow around plate explain diffraction and scattering phenomena occur in this region. The four types of imaging methods shown in Fig. 1 are at the some time our own propositions of the vectors visualization of flow waves.

a.
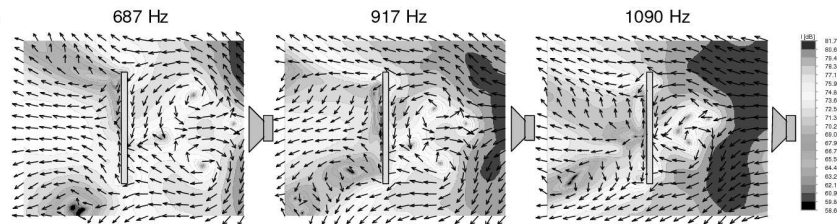

b.

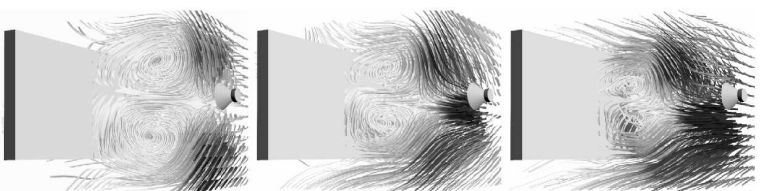

c.

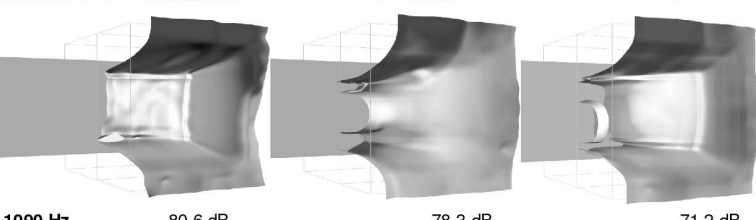

d.

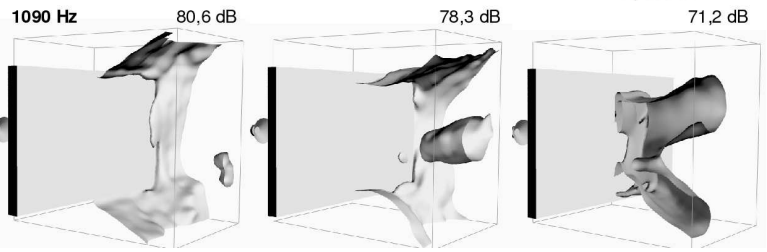

Fig. 1. Sound intensity wave distribution around hard rectangular plate (for some selected frequencies): (a) intensity map with vectors (arrows), (b) intensity streamlines in the front of plate, (c) the normal intensity component as a shape of wave in a rear side of plate, (d) intensity isosorface in the rear side space in the of plate.

In the next several experiments, a flat plate palisade of obstacles is acoustically excited by broadband noise coming from linear acoustic source (in line situated 5 loudspeakers) will be investigate. This research is an introduction to understanding the sound propagation mechanism in periodic arrays of scatterers. Investigated mod- 
els with palisades should represent very simplified rotating machines with a development of the wheel and noise propagation in arrays of scatterers region.

The approach taken here is to use direct measurement sound intensity describing the flow field around two or three-dimensional solid obstacles and illustrate the flow around them as an intensity streamlines. The study was conducted to analyze and to improve the comprehension of the mechanism of energy transfer between a turbulent flow and acoustic fields. Aerodynamic results are needed to formulate equivalent source terms while the acoustic results are needed when a coupling procedure based on acoustic boundary conditions is used. It can be expected that through the development of the proper connectivity boundary conditions between the different zones a feedback of acoustic waves from the propagation region to the source region can be incorporated.

During the tests inside-semi anechoic chamber, barriers are fixed to a hard substrate dimension $2.0 \mathrm{~m} \times 1.2 \mathrm{~m}$. The line acoustic source is about $0.5 \mathrm{~m}$ before the first barrier. All the barriers are $100 \mathrm{~mm}$ high and $1.0 \mathrm{~m}$ long, and thickness respectively: thin barriers $-2 \mathrm{~mm}$, and thick beams - $50 \mathrm{~mm}$. Cavity between barriers are $250 \mathrm{~mm}$ long (dimension of cavity: $L=250 \mathrm{~mm}$, $D=100 \mathrm{~mm}, L / D=2.5)$.

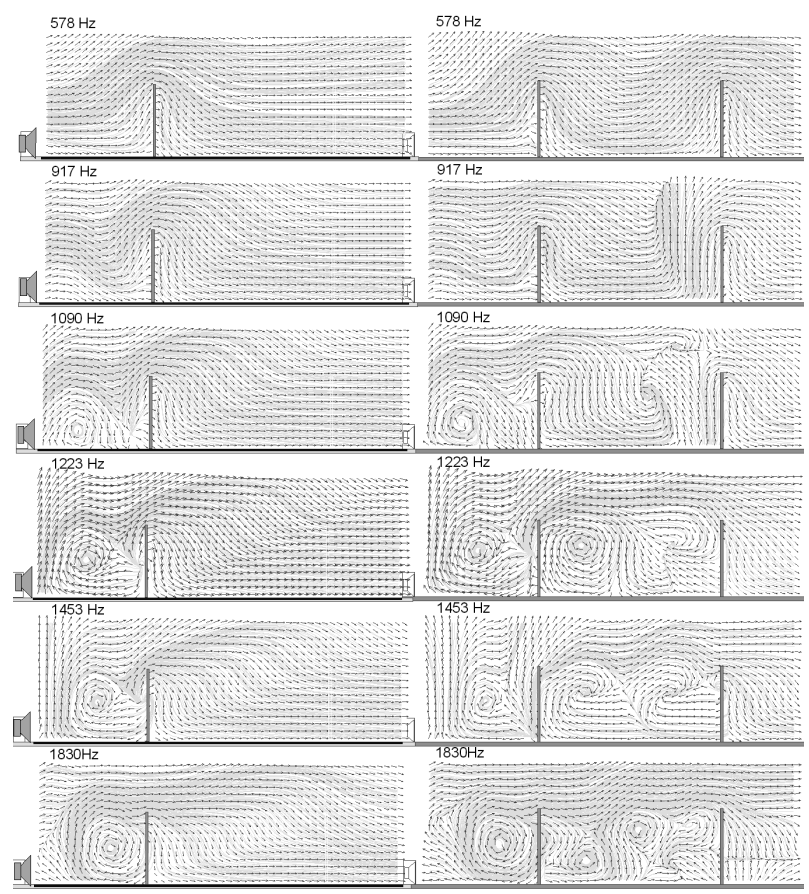

Fig. 2. Shapes of intensity streamlines describing the reaction of acoustic flow wave strike the single (left columns) end double thin barriers (right columns).

Shapes of intensity streamlines describing the reaction of acoustic flow wave strike the thin single (left columns) end double thin barriers (right columns) shown in Fig. 2. This is a comparative test what sort of reaction can we observe while a running acoustic wave hit an single ob- stacle or a series of obstacles arranged in palisades. We can show in this picture and in the rest one (Figs. 3, 4), that for the frequency band less than about $600 \mathrm{~Hz}$ we can not see a big perturbation inside the acoustic flow field, but in highness frequency region there are significant distortions of the sound intensity field in the form of vortex rotation.

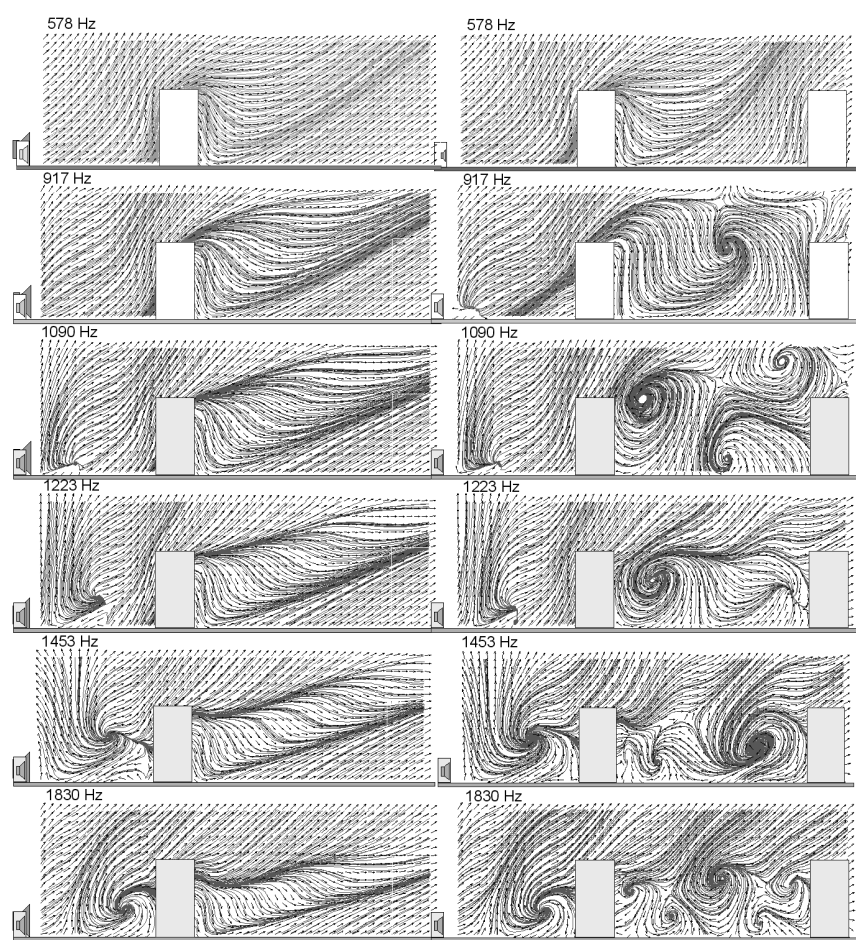

Fig. 3. The reaction of flow acoustics wave on the hard thick obstacles.

On the barrier as a broad beam (Figs. 3, 5), the trailing-edge noise mechanism is associated with the acoustic scattering of the beam turbulent boundary layer convected at the trailing edge (please remember, that top of beam is $50 \mathrm{~mm}$ long). In this processes, the quadrupolar (acoustically inefficient) nature of the convected eddies transformed into dipolar sources, which are much more acoustically efficient.

The intensity streamlines shown in Fig. 5 contains broadband components, introduced by the turbulence between palisade obstacles (four beams with cavity $250 \mathrm{~mm}$ long). The latter can be induced by two mechanisms: a wake mode mechanism, due to a periodical vortex shedding at the cavity leading edge, and a shear-layer mode mechanism, due to a feedback coupling between the flow field and the acoustic field. The complexity of many turbulent external flows in engineering equipment is considerably increased by the presence of solid walls. The key problem is that the influential turbulent eddies become progressively smaller and closer to the wall, yet these do not comply with the universality implied by Kolmogorov theory [8], because of strong shear and other in homogeneities. 


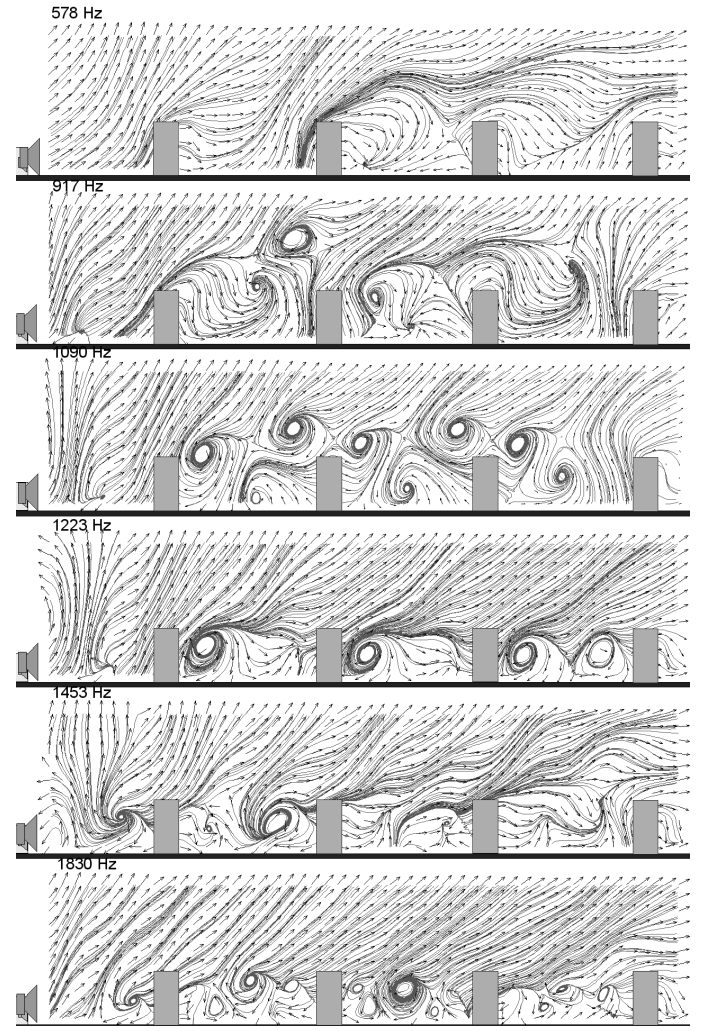

Fig. 4. The impact of flow acoustic wave on the palisades consisting of four obstacles - distribution of sound intensity streamlines vortices in the cavities (Rossiter mode).

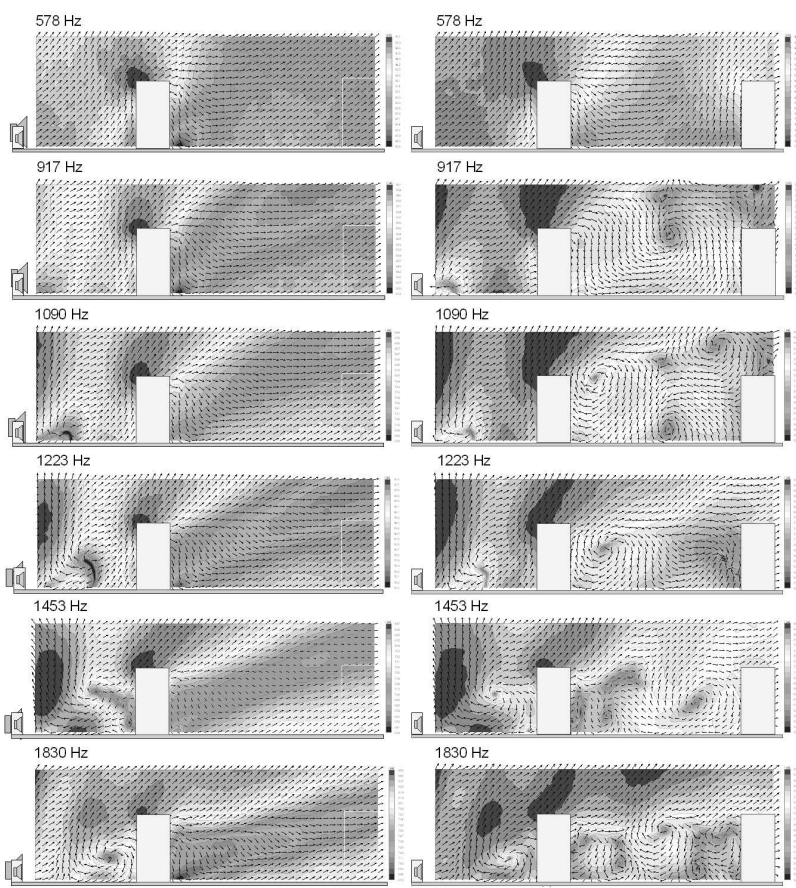

Fig. 5. The distributions sound intensity map and intensity vectors around thick obstacles.
Rossiter [10] was one of the first researchers who described the feedback mechanism based on shadow graphic observations on a number of different rectangular cavities (called "Rossiter mode"). In our research, the dominant Rossiter mode for cavity $L / D=2.5$ are excellent visible as a vortices between scaterrers (see also Figs. 2, 3). The Rossister modes are clear visible starting from frequency about $1000 \mathrm{~Hz}$ and higher. This sort of events recorded in real-live acoustics field and graphically described is a significant achievement in acoustic metrology.

\section{Some remarks about numerical aeroacoustic modeling}

Computational fluid dynamics is one of the branches of fluid mechanics that uses numerical methods and algorithms to solve and analyze problems that involve fluid flows. Computers are used to perform the millions of calculations required to simulate the interaction of fluids and gases with the complex surfaces used in engineering. However, even with simplified equations and high-speed supercomputers, only approximate solutions can be achieved in many cases.

CFD techniques are used to simulate the near-field flow, which contains the aerodynamic and nonlinear disturbance equations. Available techniques include steady RANS computations, in conjunction with stochastic models of the wave-number-frequency spectrum of the turbulence. This local flow solution has to be coupled to an acoustic numerical technique (CAA) for the prediction of far-field noise. The most practical formulations are the integral methods such as the Fflowcs-Williams-Hawkings equation [11], the boundary element method (BEM), and the Kirchhoff integral.

The CFD techniques are widely used by engineers designing or analysing devices that interact with fluid, such as vehicles, pumps, chemical apparatus or ventilation systems. The major problem faced by the CFD industry today is a shortage of skilled human resource. CFD development requires a blend of technologist and programmer. Likewise CFD analysis needs good insight in the physics of the problem, understanding of numerical methods used along with expertise in commercial CFD software packages. There are numerous commercial software packages to solve the Navier-Stokes equations. Examples of such commercial packages include the following: Ansys CFX, Fluent, Comsol, Star-CD, Abaqus, Flow-3D, and so on

CAA synergistically complement CFD by providing the user with an extended set of analytical and numerical methods to simulate the generation and transmission of sound waves through fluids. CAA prediction of narrowband and broadband noise is closely related to turbulence modelling which is central to the correct prediction of aeroacoustic sources. The sound generated by vorticity in an unbounded fluid is called aerodynamic sound. There are two principal source types in free vortical flows: a quadrupole, whose strength is determined by the unsteady Reynolds stress, and a dipole, which is important when mean mass density variations occur within 
the source region. Equivalent sources, namely dipoles (associated with aerodynamic surface pressure forcing), and quadrupoles (associated with fluctuating velocities in the flow volume), may be directly captured through transient CFD calculations [12].

The theory of aerodynamic sound was developed by Lighthill's acoustic analogy [9], which reformulated the Navier-Stokes equation into an exact, inhomogeneous wave equation whose source terms are important only within the turbulent (vortical) region. The simultaneous obtainment of the aerodynamic and acoustic field from the compressible Navier-Stokes equations in made possible by the use of very accurate algorithms and of quiet boundary conditions [3]. This can provide further insights into the noise-generation mechanism.

Lighthill was a strong advocate of the use vortex methods in fluid mechanics. It was Powell [14], however, who pioneered the interpretation of varsity as a source of sound. It is now recognized that a knowledge of the vorticity distribution in a complex flow-structure interactions not only essential for a proper understanding of mechanics of the motion, especially at low Mach numbers, but leads to the most effective means of calculating the acoustic noise produced by such flows.

A lot of a hybrid approach is usually considered for predicting aerodynamic noise [15]. The approach separates the field into aerodynamic source and acoustic propagation regions. The conventional CFD solvers are typically used to evaluate the flow-field solution in the near field to provide the aerodynamic sound source. Once the sound source is predicted, the linearized Euler Equations (LEE) or the integral methods based on Lighthill's analogy1 are used for the prediction of the acoustic wave propagation. The LEE methods assume the flow field to be a time-averaged mean flow and a time-dependent small disturbance. The extension of the near field CFD solution to the mid-field acoustic radiation can be achieved using the LEE methods [10]. However, the evaluation of the far-field radiation is prohibited due to the computer storage. The integral methods (i.e. Kirchhoff method and permeable surface Ffowcs Williams-Hawkings (FW-H) equation method), instead, predict the far-field radiating sound using the flow field quantities on a closed control surface (that encloses the entire aerodynamic source region) if the wave equation is assumed outside. The surface integration, however, has to be carried out for each far-field location. This would be still computationally intensive for a practical three-dimensional problem even though the intensity in terms of the CPU time has been much decreased compared to that required by the LEE methods. For an accurate far-field prediction, the other difficulty of using these integral methods for some aeroacoustic problems is that the control surface must completely enclose the aerodynamic source region. This may be infeasible or impossible to accomplish for some practical cases.

Most unsteady flows of technological interest are of high Reynolds number and turbulent, and the acoustic radiation is a very small by-product of the motion. The turbulence is usually product by fluid motion relative to solid boundaries or by the instability of free shear layers separating a high-speed flow (such as jet) from a stationary environment. The aerodynamic sound convert kinetic energy of rotational motions into acoustic waves involving longitudinal vibrations of fluid particles.

The complexity of CFD/CAA applications in many industrial problems, involving complex geometry and complex physics, requires the co-operation of scientists and specialists from various multi-disciplinary fields. It is expected that the activities of this working group will lead to a better overview of the capabilities and limitations of theoretical and experimental problems and developed the simulation and validation procedures.

They require the use of software with multiple capabilities and extremely high scientific knowledge inside, plus a closely connected collaboration experts: mathematicians, mechanicals and acousticians working together with one investigation group (e.g. cooperation's in a big grants).

\section{Conclusions}

In conclusion, we may set that by the direct measurement of acoustic power flow and graphically description of the results, we can explain diffraction and scattering phenomena occur on the real acoustical barriers and solved in practical way a lot of scientific and engineering problems. For instance, the flow of acoustic energy presented by the intensity streamlines shows the way of energy flow in acoustic field. Showing the paths along which the acoustic energy is transmitted may be very useful when the necessity arises to visualize the "shape of noise" radiated by vibrating mechanical structures (machines, vibrating heterogeneous plates, equipment's, etc.) and can show their acoustical activity also in limited 3D spaces. This is a form of qualitative analysis for stationary acoustic fields, which consists in a complex evaluation of the paths along which the acoustic energy of a radiating source is transported.

The application of the sound intensity technique together with $\mathrm{CFD} / \mathrm{CAA}$ methods has improved the quality of acoustic diagnostics and has made it possible to visualize energy wave phenomena (vector distribution) in a vibrating structure, or in an acoustic field around the structure. Direct energy analysis of acoustic fields was not possible earlier because the classical studies used a converter (microphone) measuring pressure changes, but pressure is a scalar element of acoustic waves. Only when direct measurements of sound intensity (e.g. as streamlines of acoustic energy - a product of acoustic pressure and acoustic particle velocity) became possible, could the wave distribution be analyzed in the form of wave acoustic energy transport.

Our experiments confirm that flow acoustic imaginations in real-live conditions are very complex, even for extremely simple models facility used in this study. These investigations may be physical understanding of acoustic wave flow phenomena in real condition measurement 
showing both qualitative and quantitative flow diagnostics. The presentation of the vector distributions of real-live acoustic fields in the areas for which it is difficult to make a theoretical analysis (direct field and near field), can explain many particulars concerning the radiation character of surface sources, and scattering of wave around obstacles. Further research can focus on the importance of these interaction phenomena for more realistic engineering applications.

Experiments in our facilities can then be designed and conducted to produce validation and verification of computational results made with common now $\mathrm{CFD} / \mathrm{CAA}$ programs [8]. This combination of theoretical and experimental testing has been used to achieve the advanced results of numerical simulations and made simulations closer to the reality.

\section{References}

[1] R.R. Dickinson, in: A Unifield Approach to the Design of Visualization Software for the Analysis of Field Problems. Proc. Three-dimensional Visualization and Display Technologies, Spie (1989) p. 173.

[2] M. Gerald-Yamasaki, Visualization of Computational Fluid Dynamics. CFD Reviue (1995).

[3] M. Hafez, et al., Computational Fluid Dynamics Review, John Wiley \& Sons, Chichester 1995.

[4] F.J. Fahy, Sound Intensity, Elsevier Applied Science, London 1989.

[5] S. Weyna, Acta Acustica. Intern. Journal on Acoustics 82, 72 (1996).

[6] C. Wagner, T. Huttl, P. Sagaut, Large-eddy simulation for acoustics. Cambridge Univ. Press (2007).
[7] D.G. Crighton, et al., Modern Methods in Analytical Acoustics. Springer-Verlag, London, (1966).

[8] M.S. Howe, J. Sound Vibr. 70, 407 (1980).

[9] M.J. Lighthill, Proceedings of the Royal Society A 211, 564 (1952).

[10] J.E. Rossiter, Wind Tunnel Experiments on the Flow over Rectangular Cavities at Subsonic and Transonic Speeds. T.R. 64037, Royal Aircraft Stablishment, (1964).

[11] J.E. Ffowcs-Williams, J. Sound Vibr. 190, 387 (1996).

[12] J.E. Ffowcs-Williams, D.L. Hawkings, Philosophical Transactions of the Royal Society 264, (1969).

[13] G. Rubio, et al., Aeroacoustic noise source mechanisms in simple expansion chamber. 12th AIAA/CEAS Conference, Cambridge, Massachusetts, p. 1, 2006.

[14] A. Powell, J. Acous. Soc. Amer. 36, 177 (1964).

[15] C.M. Mak, D.J. Oldham, The application of computational fluid dynamics to the prediction of regenerated noise in ventilation system - Inter-Noise, Newport Beach, CA, USA, p. 281 (1995).

[16] D.M. Hargreaves, H.P. Morvan, N.G. Wright, Engineering Applications of Computational Fluid Mechanics Vol. 1, 2, p. 136 (2007)

[17] W. Roeck, Hybrid methodologies for the computational aeroacoustic analysis of confined, subsonic flows, Katholieke Universiteit Leuven, Faculteit Ingenieurswetenschappen, Belgium 2007, p. 317.

[18] S. Weyna, Archiv. Acoust. 28, 191 (2003). 